Research Article

\title{
Comparison of the Stability of Two Intraocular Lenses in Primary Angle-Closure Glaucoma after Phacoemulsification
}

\author{
Chang Zhang, ${ }^{1}$ Meng Zhang $\mathbb{D}^{2},{ }^{2}$ Bing Zhang $\mathbb{D}^{1},{ }^{1}$ Shanhong Wang $\mathbb{D}^{1},{ }^{1}$ and Yuhong Wang $\mathbb{i D}^{3}$ \\ ${ }^{1}$ Eye Department, Tongde Hospital of Zhejiang Province, No. 234 Gucui Road, Hangzhou, Zhejiang Province, China \\ ${ }^{2}$ Glaucoma Department, Eye Hospital of Zhejiang Province, No. 270 West College Road, Lucheng District, Wenzhou, \\ Zhejiang Province, China \\ ${ }^{3}$ Xiamen University Affiliated Xiamen Eye Center, 336 Xiahe Road, Siming District, Xiamen, Fujian Province, China \\ Correspondence should be addressed to Yuhong Wang; wangyuhong654@163.com
}

Received 1 May 2020; Revised 20 October 2020; Accepted 3 November 2020; Published 16 November 2020

Academic Editor: Karim Mohamed Noriega

Copyright ( $) 2020$ Chang Zhang et al. This is an open access article distributed under the Creative Commons Attribution License, which permits unrestricted use, distribution, and reproduction in any medium, provided the original work is properly cited.

\begin{abstract}
Objective. To observe the stability of intraocular lenses (IOLs) in primary angle-closure glaucoma by ultralong scan depth spectraldomain optical coherence tomography (UL-OCT) after phacoemulsification. Methods. A prospective, randomized study. 73 patients (82 eyes) with primary closed-angle glaucoma and age-related cataract were included in the study. 42 eyes were implanted with ZCB00, while 40 eyes were implanted with Softec HD after phacoemulsification. The tilt, decentration, and space between IOL and posterior capsule (IOL-PC space) were analyzed using UL-OCT at 1 week, 1 month, and 3 months after surgery. The intergroup difference was compared with the paired $t$-test. Result. The difference of decentration and tilt was not statistically significant (both $P>0.05$ ) both in the horizontal and vertical positions at 1 week, 1 month, and 3 months postoperatively. The horizontal IOL-PC space is $0.111 \pm 0.091 \mathrm{~mm}^{2}, 0.044 \pm 0.066 \mathrm{~mm}^{2}$, and $0.055 \pm 0.055 \mathrm{~mm}^{2}$ in the Softec HD group and $0.458 \pm 0.488 \mathrm{~mm}^{2}, \quad 0.497 \pm 0.363 \mathrm{~mm}^{2}$, and $0.492 \pm 0.441 \mathrm{~mm}^{2}$ in the ZCB00 group. The vertical IOL-PC space is $0.102 \pm 0.061 \mathrm{~mm}^{2}, 0.037 \pm 0.052 \mathrm{~mm}^{2}$, and $0.053 \pm 0.079 \mathrm{~mm}^{2}$ in the Softec HD group and $0.692 \pm 0.815 \mathrm{~mm}^{2}, 0.510 \pm 0.415 \mathrm{~mm}^{2}$, and $0.691 \pm 0.635 \mathrm{~mm}^{2}$ in the ZCB00 group. The difference was statistically significant $(P<0.05)$ both in the horizontal and vertical positions except for the first week on the horizon. The Softec HD group is smaller than the ZCB00 group. Conclusion. There is no difference in the stability of the IOL although the IOL-PC space is different. The thickness of IOL may affect the IOL-PC space.
\end{abstract}

\section{Introduction}

Primary angle-closure glaucoma (PACG) is the most common type of glaucoma in the clinic. The anatomical structures such as shortness of the axial eye length, shallow anterior chamber, increased thickness, and curvature of the crystalline lens were different from cataract [1]. Most of the patients are combined with cataract and phacoemulsification which can relieve pupillary block. At present, there is no intraocular lens designed for glaucoma patients and no report on the selection of IOL in relevant research. However, the stability of IOL in the capsular bag is crucial for patients in visual quality [2]. The application of ultralong scan depth spectral-domain optical coherence tomography (UL-OCT) makes the state of IOL in the capsular bag clear. In this study, UL-OCT was used to measure the decentration, tilt, and IOL-PC space of IOL and analyze the factors for the better selection of IOL in the clinic.

\section{Materials and Methods}

A total of 82 eyes (73 patients) with PACG were randomly divided into 2 groups. Randomization was done using computer-generated tables (Microsoft Excel; Microsoft Corporation, Redmond, Washington). ZCB00 was performed in 42 eyes (37 patients), and Softec HD was 
performed in 40 eyes (36 patients) of patients who visited the Eye Hospital, Wenzhou Medical University, from May 2017 to May 2019 (Table 1).

Each patient has undergone a complete ophthalmological evaluation. Patients with other eye and systemic diseases, such as high myopia, uveitis, retinal diseases, corneal disease, and previous corneal or intraocular surgery, were excluded from the study. The study protocol was approved by the Zhejiang Eye Hospital Ethics Committee (another name of Zhejiang Eye Hospital is Eye Hospital, Wen Zhou Medical University) and adhered to the Declaration of Helsinki. All of the participants signed an informed consent form.

The Softec HD group was implanted with one-piece aspherical IOL (Softec HD, Lenstec, USA), which is a onepiece, biaspheric, square edge, $12 \mathrm{~mm}$ diameter, hydrophilic acrylic IOL. The ZCB00 group was implanted with one-piece aspherical IOL (ZCB00, AMO, USA), which is a plate-haptic, aspheric surface, $13 \mathrm{~mm}$ diameter, square edge hydrophobic acrylate IOL.

Phacoemulsification was performed by one experienced surgeon under topical anesthesia with a $3.2 \mathrm{~mm}$ clear corneal incision. A $4.5 \mathrm{~mm}$ capsulorhexis, centered on the dilated pupil, was performed with the aid of capsulorhexis forceps. The incision of the right eyes was on the temporal side while the left eyes on the paranasal side. The residual viscoelastic was absorbed completely.

A custom spectrometer with a unique design was developed to achieve an experimental scan depth of $7.2 \mathrm{~mm}$ in air based on the technology of spectral-domain OCT. The modification includes a transmission grating and a line scan CCD camera (Aviiva SM2 CL 2010, 2048 pixels; Atmel, San Jose, CA). $X-Y$ cross-aiming was applied to align the ULOCT scanning position necessary to image the entire IOL in the capsule and the posterior capsule (PC). The UL-OCT has an approximately $6-\mu \mathrm{m}$ axial resolution and a scan width up to $20 \mathrm{~mm}$. The accuracy and repeatability of the instrument have been confirmed in previous works $[3,4]$. The ZCB00 IOL and Softec HD IOL are shown in Figure 1.

Follow-up assessments were performed at 1 week and 1 and 3 months postoperatively. The horizontal and vertical position of IOL was performed. Then, using Image-pro plus version 6.0 , the OCT images were analyzed. The tilt angle and decentration length were measured according to the method by Alberto de Castro et al. [5, 6]. The method was based on the Scheimpflug system: IOL decentration is obtained from the distance between the IOL center and the pupillary axis. Total decentration, determined by trigonometry analysis, shows the magnitude of the result vector of horizontal and vertical decentration [7]. The tilt was calculated by dividing the slope of IOL by the slope of the limbus [8]. The OCT images were taken as in Figure 1, the curve of the surface of the IOL and the PC was drawn, and then it was substituted into the two-dimensional coordinate plane to get the scatter plot as in Figure 2. The area between the IOL and posterior capsular region was evaluated through the function of the software [9].

Statistical analyses were performed to compare the differences in the biometric data between the ZCB00 group
TABLE 1: The age and gender distribution.

\begin{tabular}{lccr}
\hline & Softec HD group & ZCB00 group & $P$ value \\
\hline Age (year) & $65.34 \pm 11.32$ & $68.25 \pm 10.68$ & $>0.05$ \\
Gender $(\mathrm{M}: \mathrm{F})$ & $13: 24$ & $11: 25$ & $>0.05$ \\
\hline
\end{tabular}

and the Softec HD group using SPSS 19.0. The Kolmogorov-Smirnov test was used to confirm the normal distribution. The tilt and decentration were compared with the paired $t$-test. The IOL-PC space was compared with the rank sum test. Differences were considered statistically significant when the $P$ value was less than 0.05 .

\section{Results}

Table 2 shows the postoperative visual acuity results in the two IOL groups. At the 3-month visit, no significant difference was found in corrected-distance visual acuity (CDVA), uncorrected-distance visual acuity (UDVA), and axial length between the groups. The thickness of IOL was significantly different between the two groups. Softec HD was thicker than ZCB00.

The total decentration is $0.182 \pm 0.054 \mathrm{~mm}$, $0.232 \pm 0.081 \mathrm{~mm}$, and $0.183 \pm 0.089 \mathrm{~mm}$ in the Softec HD group and $0.311 \pm 0.212 \mathrm{~mm}, 0.214 \pm 0.111 \mathrm{~mm}$, and 0 . $228 \pm 0.156 \mathrm{~mm}$ in the ZCB00 group at 1 week, 1 month, and 3 months postoperatively. The difference was not statistically significant.

The horizontal tilt is $1.65 \pm 6.23^{\circ}, 0.45 \pm 4.29^{\circ}$, and $-1.76 \pm 7.37^{\circ}$ in the Softec HD group and $0.05 \pm 6.21^{\circ}$, $0.34 \pm 8.51^{\circ}$, and $-0.17 \pm 6.30^{\circ}$ in the ZCB00 group at 1 week, 1 month, and 3 months postoperatively. The vertical tilt is $3.67 \pm 6.61^{\circ},-0.94 \pm 4.73^{\circ}$, and $0.54 \pm 9.51^{\circ}$ in the Softec HD group and $-0.73 \pm 7.33^{\circ}, 0.32 \pm 7.20^{\circ}$, and $0.07 \pm 5.52^{\circ}$ in the ZCB00 group. The difference was not statistically significant both in the horizontal and vertical positions.

Table 3 shows the horizontal IOL-PC space in the two study groups. The difference was statistically significant $(P<0.05)$ except for the first week. Table 4 shows the vertical IOL-PC space. The difference was statistically significant $(P<0.05)$. The IOL-PC space in the Softec HD group is smaller than the space in the ZCB00 group (Figure 3).

\section{Discussion}

The quality of visual acuity will be improved without decentration and tilt [10]. However, a previous study had reported that IOL tilt was more extensive in the eyes with glaucoma than normal cataract [11]. The increased thickness, curvature of the crystalline lens, and a more anterior lens position indicate that lens capsule configuration is also abnormal in eyes with angle-closure glaucoma. It is therefore reasonable to conclude that the implanted IOL is apt to be tilted or decentered in eyes with CAG. Another study found $\mathrm{AL}$ is negatively correlated with crystalline lens tilt, which means IOLs are more prone to tilt in patients with a short AL [12]. The possible explanation is that the crystalline lens is more likely to tilt in the crowded intraocular space. In addition, Chen et al. [13] have found that the thicker lens was 


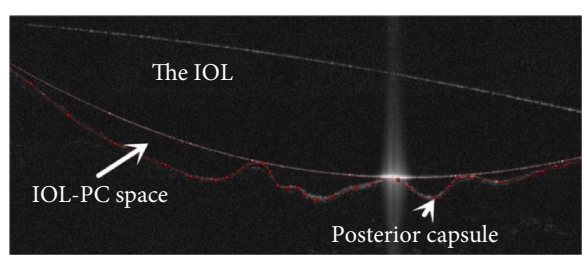

(a)

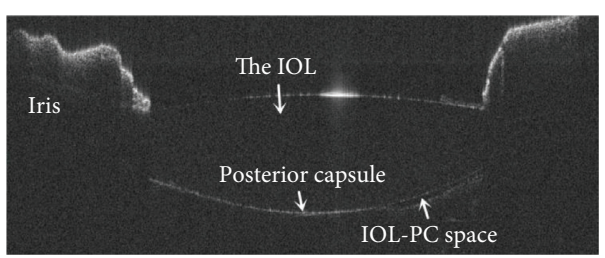

(b)

Figure 1: The UL-OCT detects the boundaries of intraocular lens (IOL), posterior capsule (PC), and the space between IOL and posterior capsule (IOL-PC space). (a) The ZCB00 IOL. (b) The Softec HD IOL. UL-OCT =ultralong scan depth spectral-domain optical coherence tomography.

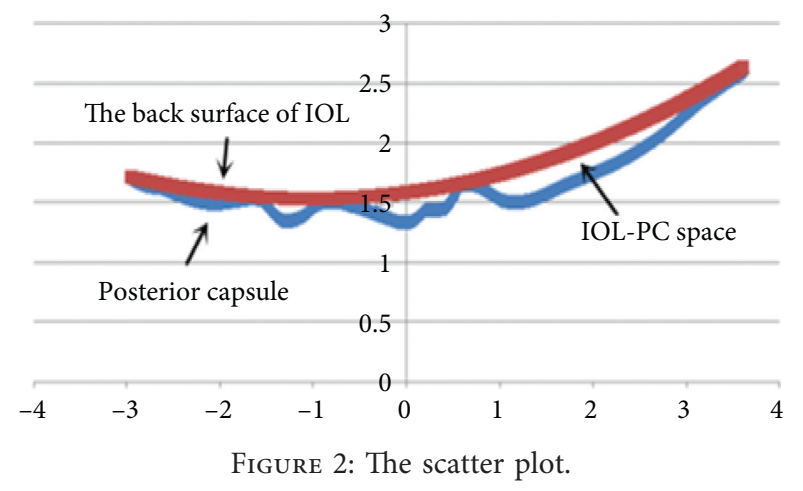

TABLE 2: Postoperative results in the two study groups.

\begin{tabular}{lccc}
\hline & Softec HD & ZCB00 & $P$ \\
\hline UDVA (logMAR) & $0.18 \pm 0.13$ & $0.21 \pm 0.09$ & 0.28 \\
CDVA (logMAR) & $0.10 \pm 0.09$ & $0.13 \pm 0.12$ & 0.24 \\
Axial length (mm) & $22.84 \pm 1.92$ & $22.92 \pm 2.11$ & 0.32 \\
Thickness of IOL (mm) & $1.47 \pm 0.355$ & $1.19 \pm 0.287$ & 0.03 \\
\hline
\end{tabular}

CDVA = corrected-distance visual acuity; UDVA = uncorrected-distance visual acuity; $\log \mathrm{MAR}=\log$ arithm of the minimum angle of resolution.

TABLE 3: Horizontal IOL-PC space $\left(\mathrm{mm}^{2}\right)$ postoperative.

\begin{tabular}{lcccc}
\hline & 1 week & 1 month & 3 months & $P$ value \\
\hline $\begin{array}{l}\text { Softec } \\
\left(\mathrm{mm}^{2}\right)\end{array}$ & $0.11 \pm 0.091$ & $0.044 \pm 0.066$ & $0.055 \pm 0.055$ & 0.189 \\
$\begin{array}{l}\mathrm{ZCB} 00 \\
\left(\mathrm{~mm}^{2}\right)\end{array}$ & $0.458 \pm 0.488$ & $0.497 \pm 0.363$ & $0.492 \pm 0.441$ & 0.823 \\
$P$ value & 0.15 & 0.002 & 0.001 & \\
\hline
\end{tabular}

TABLE 4: Vertical IOL-PC space $\left(\mathrm{mm}^{2}\right)$ postoperative.

\begin{tabular}{lcccc}
\hline & 1 week & 1 month & 3 months & $P$ value \\
\hline $\begin{array}{l}\text { Softec } \\
\left(\mathrm{mm}^{2}\right)\end{array}$ & $0.102 \pm 0.061$ & $0.037 \pm 0.052$ & $0.053 \pm 0.079$ & 0.035 \\
$\begin{array}{l}\mathrm{ZCB} 00 \\
\left(\mathrm{~mm}^{2}\right)\end{array}$ & $0.692 \pm 0.815$ & $0.510 \pm 0.415$ & $0.691 \pm 0.635$ & 0.997 \\
$P$ value & 0.013 & 0.001 & 0.006 & \\
\hline
\end{tabular}

strongly correlated with larger decentration. Therefore, the decentration and tilt in PACG may be different from cataract, so the research may have a reference value for the choice of IOL in clinical work.
We have found that the IOL-PC space of Softec HD, which is constituted by hydrophilic acrylate, is significantly smaller than that of ZCB00 made of hydrophobic acrylate material. The finding is different from some research: hydrophobic acrylates have better biocompatibility, which can make the optical part of IOL close to the posterior capsule and reduce the incidence of posterior cataract and the shrinkage of the capsule. However, the hydrophilic acrylate material could not be closely attached to the posterior capsule, which provided space for LECs growth and proliferation [9]. The reasons may be as follows: the thickness of IOL is determined by the material and the diopter. In this experiment, the average thickness of Softec HD is $1.47 \pm 0.355 \mathrm{~mm}$ and ZCB00 is $1.19 \pm 0.287 \mathrm{~mm}$. The difference is statistically significant. If the IOL is assumed to be a standard ellipsoid, according to the ellipse volume formula $V=4 / 3 \pi a b c(a, b$, and $c$, respectively, represent half of each axis), where the length of $a$ and $b$ takes the radius of the optical surface and $c$ takes half of the thickness, then it can be inferred that the volume of Softec HD is about $50.87 \pm 12.28 \mathrm{~mm}^{3}$ and ZCB00 is about $44.83 \pm 10.81 \mathrm{~mm}^{3}$. Takuhei et al. have reported that the lens thickness is positively correlated with axial length [14]. Another study found that equatorial capsular bag diameter is correlated with axial length [15]. A significant difference in axial length was not found between the study groups, which means a difference in capsular bag size is not a likely reason for the larger IOLPC space observed in the ZCB00 group. Then, we can speculate that Softec HD with a larger volume fills the capsular bag better. As a result, the IOL-PC space in Softec $\mathrm{HD}$ is smaller than ZCB00.

The IOL-PC space on visual function is in agreement with the concept "no space, no cell, no PCO" [16]. Studies have reported that, with increasing severity of PCO, visual acuity and stray light deteriorate [17]. In our study, a thicker IOL could promote the elimination of IOL-PC space. So, we can infer that the incidence of posterior cataract is reduced and vision will be maintained better with a thicker IOL.

There was no statistical difference between the tilt and decentration although the IOL-PC space was significantly different. The result is similar to the research of Katayama et al. [18]: decentration and tilt did not change significantly although the posterior capsular opacity (PCO) value in the hydrophilic group increased significantly with time and was statistically significantly higher than in the hydrophobic group at 18 and 24 months postoperatively in either group. 


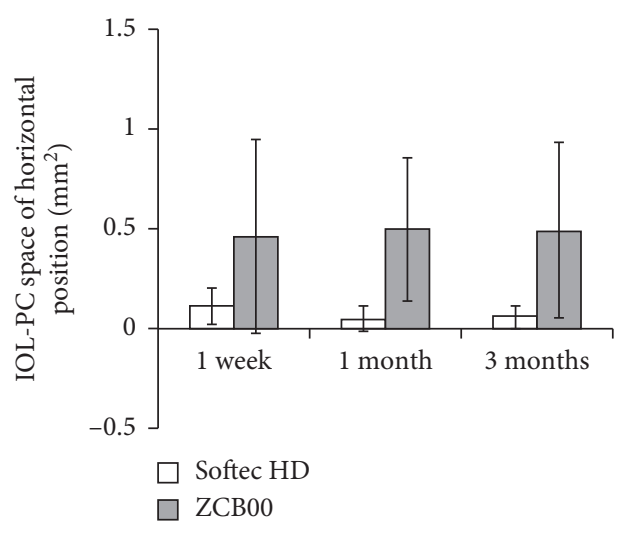

(a)

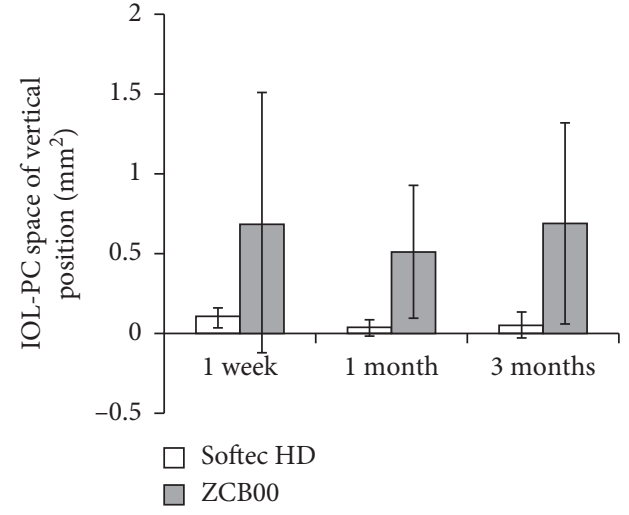

(b)

Figure 3: Postoperative IOL-PC space (SD) at different time points. Significant difference was found between the horizontal and vertical positions when compared at 1 week, 1 month, and 3 months postoperatively $(P<0.05)$ except for the first week on the horizon.

The reasons may be as follows: first, Modesti et al. [19] considered that the maximum diameter of the lens capsule at any time after cataract surgery was $<12.0 \mathrm{~mm}$, which was smaller than that of the IOLs in this study $(12 \mathrm{~mm}$ in Softec $\mathrm{HD}$ and $13 \mathrm{~mm}$ in ZCB00). But, the lens capsule is thicker than the intraocular lens, so the IOL is stuck in the center of the capsule without bound to the posterior capsule tightly. As a result, haptic provides most of the strength for the stability of the IOL. This finding explains that IOLs with a diameter greater than $12 \mathrm{~mm}$ were more stable than IOLs with a diameter less than $12 \mathrm{~mm}$ [20]. Besides, the two IOL's haptics are made of acrylate, which is soft and firm. When the capsule is contracted, the soft haptic can be compressed independently without transmitting this force to the IOL optics. Therefore, in the case of different IOL-PC space, stability is less affected.

The limitations of this trial were that it was limited by clinical work and only followed up for 3 months. PCO begins three months after surgery, and the long-term stability of IOL in patients with PACG remains unknown. Besides, studies have shown that mathematics can be used based on cross-sectional and sagittal images. Modeling restores the $3 \mathrm{D}$ stereo image of the IOL to accurately calculate the maximum tilt and decentration of the IOL [21]. But in clinical work, mathematical modeling requires a lot of time and effort.

In summary, by using UL-OCT, we could clearly observe the IOL and posterior capsule. The objective quantification of thickness demonstrated a correlation with IOL-PC space. Maybe the haptic and diameter of IOL length play a more important role in stability than IOL-PC space. These results suggested that UL-OCT could be used as a powerful method to evaluate the IOL-PC space and the tilt and decentration of IOL.

\section{Data Availability}

The data that support the findings of this study are available from the corresponding author upon reasonable request.

\section{Disclosure}

The authors alone are responsible for the content and writing of the paper.

\section{Conflicts of Interest}

The authors report no conflicts of interest.

\section{Acknowledgments}

This work was funded by the Key Laboratory of Myopia, Health Commission, Fudan University.

\section{References}

[1] R. F. Loew, "Aetiology of the anatomical basis for primary angleclosure glaucoma. Biometrical comparisons between normal eyes and eyes with primary angle-closure glaucoma," British Journal of Ophthalmology, vol. 54, no. 3, pp. 161-169, 1970.

[2] R. N. McNeely, E. Pazo, A. Spence et al., "Visual quality and performance comparison between 2 refractive rotationally asymmetric multifocal intraocular lenses," Journal of Cataract and Refractive Surgery, vol. 43, no. 8, pp. 1020-1026, 2017.

[3] M. Shen, L. Cui, M. Li, D. Zhu et al., "Extended scan depth optical coherence tomography for evaluating ocular surface shape," Journal of Biomedical Optics, vol. 16, no. 5, Article ID 056007, 2011.

[4] M. Shen, L. Cui, C. Riley, M. R. Wang, and J. Wang, "Characterization of soft contact lens edge fitting using ultrahigh resolution and ultra-long scan depth optical coherence tomography," Investigative Opthalmology and Visual Science, vol. 52, no. 7, pp. 4091-4097, 2011.

[5] P.-Y. Chang, C.-Y. Lian, J.-K. Wang, P.-Y. Su, J.-Y. Wang, and S.-W. Chang, "Surgical approach affects intraocular lens decentration," Journal of the Formosan Medical Association, vol. 116, no. 3, pp. 177-184, 2017.

[6] A. D. Castro, P. Rosales, and S. Marcos, "Tilt and decentration of intraocular lenses in vivo from Purkinie and Scheimpflug imaging Validation study," Journal of Cataract and Refractive Surgery, vol. 33, no. 3, pp. 418-429, 2007.

[7] K. Kránitz, K. Miháltz, G. L. Sándor, A. Takacs, M. C. Knorz, and Z. Z. Nagy, "Intraocular lens tilt and decentration 
measured by Scheimpflug camera following manual or femtosecond laser-created continuous circular capsulotomy," Journal of Refractive Surgery, vol. 28, no. 4, pp. 259-263, 2012.

[8] D. A. Kumar, A. Agarwal, G. Prakash, S. Jacob, Y. Saravanan, and A. Agarwal, "Evaluation of intraocular lens tilt with anterior segment optical coherence tomography," American Journal of Ophthalmology, vol. 151, no. 3, pp. 406-412, 2011.

[9] S. Yu, C. Lu, and X. Tang, "Application of spectral domain optical coherence tomography to objectively evaluate posterior capsular opacity in vivo," Journal of Ophthalmology, vol. 2018, Article ID 5461784, 9 pages, 2018.

[10] T. Lawu, K. Mukai, H. Matsushima, and T. Senoo, "Effects of decentration and tilt on the optical performance of 6 aspheric intraocular lens designs in a model eye," Journal of Cataract and Refractive Surgery, vol. 45, no. 5, pp. 662-668, 2019.

[11] K. Hayashi, H. Hayashi, F. Nakao, and F. Hayashi, "Intraocular lens tilt and decentration after implantation in eyes with glaucoma," Journal of Cataract and Refractive Surgery, vol. 25, no. 11, pp. 1515-1520, 1999.

[12] L. Wang, R. G. De Souza, M. P. Weikert, and D. D. Koch, "Evaluation of crystalline lens and intraocular lens tilt using a swept-source optical coherence tomography biometer," Journal of Cataract and Refractive Surgery, vol. 45, no. 1, pp. 35-40, 2019.

[13] X. Chen, X. Gu, W. Wang et al., "Characteristics and factors associated with intraocular lens tilt and decentration after cataract surgery," Journal of Cataract and Refractive Surgery, vol. 46, no. 8, pp. 1126-1131, 2020.

[14] T. Shoji, N. Kato, S. Ishikawa et al., "Association between axial length and in vivo human crystalline lens biometry during accommodation: a swept-source optical coherence tomography study," Japanese Journal of Ophthalmology, vol. 64, no. 1, pp. 93-101, 2020.

[15] M. Tehrani, B. H. Dick, F. Krummenauer, G. Pfirrmann, T. Boyle, and B. M. Stoffelns, "Capsule measuring ring to predict capsular bag diameter and follow its course after foldable intraocular lens implantation," Journal of Cataract and Refractive Surgery, vol. 29, no. 11, pp. 2127-2134, 2003.

[16] D. J. Apple, K. D. Solomon, M. R. Tetz et al., "Posterior capsule opacification," Survey of Ophthalmology, vol. 37, no. 2, pp. 73-116, 1992.

[17] M. C. J. van Bree, T. J. T. P. van den Berg, and B. L. M. Zijlmans, "Posterior capsule opacification severity, assessed with straylight measurement, as main indicator of early visual function deterioration," Ophthalmology, vol. 120, no. 1, pp. 20-33, 2013.

[18] Y. Katayama, S. Kobayakawa, H. Yanagawa, and T. Tochikubo, "The relationship between the adhesion characteristics of acrylic intraocular lens materials and posterior capsule opacification," Ophthalmic Research, vol. 39, no. 5, pp. 276-281, 2007.

[19] M. Modesti, G. Pasqualitto, R. I. Appolloni, and P. Sourdille, "Preoperative and postoperative size and movements of the lens capsular bag: ultrasound biomicroscopy analysis," Journal of Cataract and Refractive Surgery, vol. 37, no. 10, pp. 1775-1784, 2011.

[20] B. C. Pecorella, R. Khoramnia, L. F. Weber, T. Tandogan, and G. U. Auffarth, "Rotation and decentration of an undersized plate-haptic trifocal toric intraocular lens in an eye with moderate myopia," Journal of Cataract and Refractive Surgery, vol. 42, no. 3, pp. 489-493, 2016.

[21] L. Lin, W. Ke, Y. Yan et al., "Calculate method of the IOL tilt and decentration based on surface fitting," Beijing Biomedical Engineering, vol. 32, no. 2, pp. 164-168, 2013. 